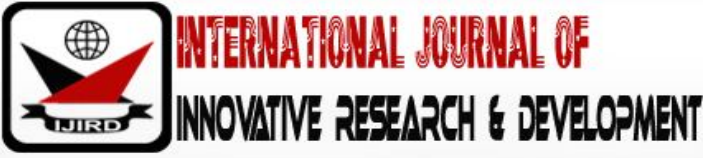

ISSN 2278 - 0211 (Online)

\section{Rapid Immunochromatographic Test (RDT) Versus ELISA Technique for Diagnosing Toxoplasmosis among Individuals with Mental Disabilities}

\author{
Faika Hassaneina \\ Lecturer, Department of Microbiology and Immunology, Phros University in Alexandria, Egypt \\ Amany I. Shehata \\ Lecturer, Department of Tropical Health, Parasitology and Medical Entomology High Institute of \\ Public Health, Alexandria University, Egypt
}

\begin{abstract}
:
Background: Toxoplasmagondii is a ubiquitous protozoan parasite that is estimated to infect one-third of the world's human population. In the quest for a suitable diagnostic tool for detection of T. gondii antibodies, the rapid immunochromatographic test has been validated versus the enzyme linked immunosorbent assay (ELISA) technique for diagnosing toxoplasmosis among individuals with mental disabilities. Method: A cross-sectional study was carried out in Alexandria governorate, Egypt during the period from December 2015 to May 2016. Blood samples were collected from 188 mental disabled individuals. The first aliquot was tested by the rapid diagnostic test (RDT), the 2nd and 3rd aliquots were tested quantitatively for anti T. gondiilgG and IgM antibodies using ELISA test. Results: The seroprevalence of toxoplasmosis based on RDT was $26.6 \%$, it significantly increased to $48.9 \%$ by using ELISA. The diagnostic parameters of RDT versus ELISA for IgG were: sensitivity $54.4 \%$, specificity $100 \%$, PPV $100 \%$, NPV $69.6 \%$ with a diagnostic efficiency of $77.7 \%$, while for IGM were: sensitivity $29.03 \%$, specificity $100 \%$, PPV $100 \%$, NPV $87.7 \%$ and diagnostic efficiency $88.3 \%$. Cohen's kappa coefficient was 0.549 and 0.406 for IgG and IgM respectively indicating good to moderate agreement between the two tests for detecting IgG and poor to fair agreement for IgM. Conclusion: Although RDT is inferior compared to ELISA, its speed, simplicity, low cost, high specificity and its good diagnostic efficiency in detecting IGG make it an important preliminary screening tool for diagnosing toxoplasmosis in remote endemic areas where facilities for ELISA are not available.
\end{abstract}

Keywords: Rapid Immunochromatographic Test (RDT), Enzyme Linked Immunosorbent Assay (ELISA) technique, toxoplasmosis, mental disabilities

\section{Introduction}

Toxoplasma gondii is a unicellular coccidian parasite with a worldwide distribution. Man and other warm blooded animals act as intermediate hosts while members of Felidae family are actually the definitive host (Monocada, \& Montoya; Robert-Gangneux, \& Dardé, 2012). About 25-30\% of the human population is found to be infected by T. gondii worldwide (Montoya, \& Liesenfeld, 2004). The prevalence varies widely between countries (10-80\%) and often within a given country or between different socioeconomic strata of a specific population (Pappas, Roussos, \& Falagas, 2009; Rosso, et al., 2008).

The majority of human transmission is caused either by ingestion of tissue cysts in infected meat or ingestion of sporulated oocyst from contaminated soil, water, food or directly from feline feces (Robert-Gangneux, \& Dardé, 2012). Transplacental infection, following maternal infection with T. gondii for the first time during gestation, is also an important way of transmission (SYROCOT, Thiébaut, Leproust, Chêne, and Gilbert, 2007). Other routes of transmission have been documented including organ transplantation or laboratory accidents (Monocada, \& Montoya, 2012). Most cases of toxoplasmosis are asymptomatic or may suffer from influenza like symptoms. Immunocompromized patients usually develop fulminating, life threatening complications as pneumonia and encephalitis (Luft, \& Remington, 1992). Primary infection during pregnancy may cause spontaneous abortion, still birth or congenital toxoplasmosis with ocular and neurological manifestations (Monocada, \& Montoya, 2012; Elsheikha, 2008).

Several laboratory tests are available for the diagnosis of T. gondii including: serological tests, PCR, histological and cytological examination of tissue and body fluids (Monocada, \& Montoya, 2012). A variety of serological tests have been developed to detect different antibody classes or antigens (Liu, Wang, Huang \& Zhu, 2015). The most used assay today is the indirect enzyme linked immunosorbent assay (ELISA) for routine detection of specific anti-toxoplasma IgG and IgM antibodies 
(Robert-Gangneux, \& Dardé, 2012). As the presence of specific IgG antibodies just implies the parasite infection with no information on infection time and as IgM antibodies isn't an accurate marker for acute infection (Meek, Diepersloot, van Gool, Speijer \& Peek, 2003), IgG avidity was described by Hedman et al (1989) (Hedman, Lappalainen, Seppäiä \& Mäkelä, 1989). The variation in the antibody binding strength was evaluated by ELISA to differentiate between acute and chronic T. gondii infection (Robert-Gangneux, \& Dardé, 2012, Lappalainen et al., 1993). Though ELISA tests are sensitive and specific, yet they are expensive, require a specialized laboratory and expert personnel (Liu, Wang, Huang \& Zhu, 2015).

The immunochromatography test (ICT) is a rapid diagnostic test (RDT) in which the colloidal gold labeled antigen or antibody is used as the tracer and the cellulose membrane is used as the solid support. Antibodies or antigens looked for are placed at the sample pad on the nitrocellulose membrane. They will slowly infiltrate the pad by a capillary action. The antigen antibody reaction will be detected as a colloidal color reaction (Wang et al., 2011). As RDT is easy to perform, rapid and doesn't need special equipment, nor expert personals, so it is suitable for field application (Liu, Wang, Huang \& Zhu, 2015). Our study is planned to assess the validity of a commercially available RDT versus the conventionally used ELISA for the diagnosis of toxoplasmosis among mentally disabled individuals.

\section{Materials and Methods}

\subsection{Study Design, Study Population and Sample Collection}

A cross-sectional study was carried out in different rehabilitation centers for mental disabilities representing different sectors in Alexandria governorate, Egypt, during the period from December 2015 to May 2016. Venous blood samples were collected from 188 individuals aged from 3 to 37 years and whose parents accepted their participation in the study. Samples were transferred to the parasitological laboratory of High Institute of Public Health for processing. The serum was separated from the whole blood by centrifugation at 3,000 rpm for 10 minutes at room temperature, then divided into three aliquots and stored at $-20^{\circ}$ Cuntil use.

\subsection{Serological Testing}

The first aliquot was tested by using a rapid diagnostic test kit "On-site toxoIgG/ IgM lateral flow chromatography assay". The assay could detect and differentiate between IgG and IgM antibodies in serum. The $2^{\text {nd }}$ and $3^{\text {rd }}$ aliquots were tested quantitatively for anti - Toxoplasma gondii IgG and IgM antibodies respectively using ELISA test, strictly following manufacturer's instructions. The results were read at $450 \mathrm{~nm}$ by a micro-well reader compared in a parallel manner with calibrator and controls. All results at or above cut off value (1.169 for IgG and 1.15 for IgM) were considered positive.

\subsection{Statistical analysis}

Correlation of the diagnostic parameters of RDT versus ELISA as a gold standard technique was done using diagnostic accuracy tests (Simuudic, 2009). Chi-square and Cohen's Kappa coefficient were used for comparison between variables, pvalues $\leq 0.05$ was considered statistically significant.

\subsection{Ethical Consideration}

Ethical approval was obtained from the Ethics Review Committee of the High Institute of Public Health, Alexandria University, Egypt. Informed written consent was either signed or thumb-printed from parents or guardians of the children accepting to be involved in the study before its start. Approval was also obtained from the Vocational Rehabilitation Managers and the Social Affairs Directorate.

\section{Results}

The seroprevalence of toxoplasmosis among 188 mentally disabled individuals examined based on RDT was $26.6 \%$, it significantly increased to $48.9 \%$ when using ELISA technique. Also, IgMantibodies were detected in $4.8 \%$ of them by RDT and significantly increased to $16.5 \%$ by ELISA test (Table 1). As regards distribution of anti-Toxoplasma specific IgG and IgM antibodies among examined sample, it was found that individuals who were $\operatorname{IgG}(+\mathrm{ve}) \mathrm{EIgM}(-\mathrm{ve})$ which indicated a previous exposure or chronic infection (immune cases) were (32.45\%) and (21.8\%) as detected by ELISA and RDT respectively. Positive results for both IgG\&IgM indicating either primary infection or reactivation of chronic infection (probable acute infection) were $(16.5 \%) \&(4.8 \%)$ for ELISA and RDT respectively while negative results for both antibodies (susceptible cases) were $(51.1 \%)$ and $(73.4 \%)$ respectively (Table 2 ).

All cases reported positive by RDT for IgM and IgG were also positive by ELISA. However, 42 cases that were positive for IgG by ELISA test were found negative by RDT. The same was also seen for IgM as 22 positive cases by ELISA test were negative by RDT. Cohen's kappa coefficient was 0.549 and 0.406 for IgG and IgM respectively indicating a good to moderate agreement between RDT and ELISA in detecting IgG and a poor to fair agreement in detecting IgM, (Table 3, 4). By computing the diagnostic parameters of RDT versus ELISA, it was evident that RDT had a sensitivity of $54.4 \%$, a specificity of $100 \%$, PPV of $100 \%$, NPV of $69.6 \%$ with a diagnostic efficiency of $77.7 \%$ for IgG. As regards IgM, the sensitivity was $29.03 \%$, the specificity was $100 \%$, PPV was $100 \%$, NPV was $87.7 \%$ and the diagnostic efficiency was $88.3 \%$ (Table 5).

\section{Discussion}


Toxoplasmosis is a major public health problem that has a high socioeconomic impact resulting mainly from the cost of caring for sick, mentally retarded and blind children (Roberts, Murrell \& Marks, 1994). It is also responsible for significant morbidity and mortality especially in congenitally infected and immunocompromized individuals (Elsheikha, 2008; Luft, \& Remington, 1992). So, continuous and detailed epidemiological surveillance is required to estimate its risk of infection. Accurate diagnostic assays for detection of $\mathrm{T}$. gondii are crucial as a step toward management and control of the disease. During the past few years, there has been an increased interest in the diagnosis using techniques which are rapid, simple and inexpensive as well as sensitive and specific to be used either in field surveys or in outpatient clinic (Nourollahi, Shad-Del, Hosseini \& Dinani,2004). The present study is meant to standardize the commercially available rapid immunochromatographic technique (RCT) for qualitative detection of IgG and IgM specific antibodies against T. gondii.

In the present study, the prevalence rates of anti-Toxoplasma IgG (48.9\%) and IgM (16.5\%) using ELISA test, are in line with those reported among different categories of the Egyptian population (El-Nahas, El-Tantawy, Farag \& Alsalem, 2014; Tammam, 2013; El Deeb, Salah-Eldin, Khodeer \& Allah, 2012; Elsheikha, 2009; ). But, IgG rate is lower than what was recorded by El Nahas et al (2014) (76.9\%- 92.6\%) (El-Nahas, El-Tantawy, Farag \& Alsalem, 2014). While IgM rate is much higher than what was reported by El Deeb et al (2.8\%) (El Deeb, Salah-Eldin, Khodeer \& Allah, 2012).

In the present work, the IgG seroprevalence using RDT is $26.6 \%$, which is almost comparable with what was recorded in Saudi Arabia among pregnant woman using the same technique (21.3\%) (Imam, Azzam \& Attia 2016), but it is higher than the seroprevalence recorded in Sri Lanka(12.3\%) (Chandrasena et al., 2016). It is lower than what was recorded among pregnant women in West Africa (48.5\%) (Ogouyèmi-Hounto et al., 2014) and what was reported among the Residents of Seokmo-do (Island) in Korea (29.2- 45.3\% from 2010 to 2013) (Kim et al., 2017). Negative results for both IgG and IgM in the present study using RDT was 73.4\% which is similar to what was reported in Saudi by Imam et al (78.7\%) but lower than what was reported in Sri Lanka (87.7\%) (Imam, Azzam \& Attia; Chandrasena et al., 2016). In contrast to our results these two studies didn't detect IgM antibodies among the studied samples. The study done on individuals with severe mental illness (psychotic disorders) attending the Federal Psychiatric hospital, Benin, Nigeria revealed that $30.7 \%$ and $7.14 \%$ of the cases were Toxoplasma IgGand IgM positive respectively (James, Agbonile, Okolo, Lawani \& Omoaregba, 2017).

The obtained results of the diagnostic parameters of RDT versus ELISA technique as gold standard revealed that RDT could detect about half the individuals having specific IgG antibodies while it could detect only $29 \%$ of those having IgM antibodies (sensitivity was $54.35 \%$ and $29.03 \%$ respectively). On the other hand, RDT could exclude all individuals who were not infected (specificity was 100\%). All IgG and / or IgM positive cases detected by RDT were actually infected with T. gondii (PPV 100\%) while $69.6 \%$ who were IgG negative and $87.7 \%$ of those who were IgM negative by RDT were actually not infected (NPV were $69.6 \%$ and $87.7 \%$ respectively).

This study also revealed a good to moderate agreement between the two tests for detecting IgG antibodies (77.7\% with kappa test of 0.549). As regards IgM, instead that the total agreement was 88.3\%, Kappa test was 0.406 indicating poor to fair agreement. This was due to the low agreement percentage among positive cases $(29.03 \%)$.

In accordance to our findings, the study done on healthy blood donors from blood transfusion center of Military hospital, Omdurman, Sudan, revealed that ELISA technique was more sensitive than RDT in detecting IgG positive sera (27.15\% vs 18.8\%) (Abdelkareem, and Eltayeb, 2015). On the other hand, a study was done in Baghdad on female visitors to a private gynecology clinic to compare the results of Toxoplasma IgG and IgM rapid test to those of ELISA test . RDT showed a sensitivity of $88 \%$ and a specificity of $89.29 \%$ when detecting IgG, while it revealed a sensitivity of $95 \%$ and a specificity of 98.9\% for IgM (Hassan, Ghazi \& Ahmed, 2010). The study done on pregnant women in Benin aimed to evaluate RDT versus ARCHITECT CIMA technique. Performances for IgG were: sensitivity 97\%, specificity 100\%, PPV 100\%, NPV 97.1\% while those for IgM were: sensitivity 33.3\%, specificity 100\%, PPV 100\%, NPV 99.2\% (Ogouyèmi-Hounto et al., 2014).

Most commercial serological kits use native antigens prepared from tachyzoites (whole cell lysate Ag). These tests are difficult to standardize and frequently provide insufficient specificity (Wilson et al., 1997). Recently, some studies tried to improve the performance of RDT by experimentally testing different purified recombinant protein antigens of T. gondii (surface antigens SAG, dense granule antigens GRA, rhoptry antigens and matrix proteins) either on animal or human sera (Chong et al., 2011; Huang et al., 2004)). These antigens were previously and widely used in ELISA assays for routine diagnosis and were documented to improve its serological diagnosis of toxoplasmosis (Holec-Gasio \& Kur; Kotresha \& Noordin, 2010).

Song et al developed a RDT to diagnose T. gondii specific antibodies in human, by linking a recombinant antigenic N-terminal half of SAG1 with an intrinsically unstructured domain of $\mathrm{GRA}_{2}$ protein. The overall specificity and sensitivity were estimated as $100 \%$ and $97.1 \%$ respectively when compared with ELISA results (Song et al., 2013). Kim et al compared and analyzed results of ELISA and RDT (mounted with recombinant fragment of SAG1 antigen) using the sera collected from residents of Gyodong-do (Island), Korea. The sero-positivity recorded by ELISA was ranging between $14.7 \%$ and $32.1 \%$. Meanwhile RDT, more sensitively, detected a higher prevalence range from $21.7 \%$ to $33.1 \%$. ELISA values agreed fairly well with RDT results when the OD was either under 0.15 or above 0.35 . Disagreement between the results of the two tests appeared when the OD ranged from 0.15 to 0.35 (around the cut off value: 0.25 ). As at the range of 0.15 to 0.24 , RDT was able to detect $16.1 \%$ more positive cases that might represent an early or acute toxoplasmosis which could be missed by ELISA. On the other hand, RDT agreed with ELISA only in $64.1 \%$ of cases at the OD range of $0.25-0.34$. This could be interpreted either as the ELISA at this range was showing a false positive rate of $35.9 \%$ or as RDT having a low detection rate, so ELISA was able to detect $35.9 \%$ more positives (most probably chronic infections). Abundant antibodies against various Toxoplasma antigens are usually 
found during chronic infections. These antigens could be detected by ELISA as the test used T. gondii whole cell lysate as antigen. On the other hand, RDT results would be limited as it used only rSAG1A-GRA2 antigen (Kim et al., 2017).

In conclusion, although the RDT performance is found to be inferior compared to ELISA especially in detecting IgM, yet it has high specificity (100\%) so we can recommend it for excluding those having the disease ( detect the absence of the disease). Also, its speed, simplicity and low cost together with its good-moderate performance in detecting IgG, present an important diagnostic potential for RDT in clinical practice providing a preliminary screening tool to detect IgG antibodies for routine tests and mass screening programs in areas (remote ones) where toxoplasmosis is endemic, where laboratory facilities for ELISA and expert technicians are not usually available. Positive cases should be re-examined by a more sensitive test to detect early infection (as by IgG avidity test). Also, the development of a commercial RDT based on more sensitive and specific antigens is mandatory for the diagnosis and the discrimination of IgM and IgG antibodies, which is a crucial step towards management and control of the disease.

\section{Acknowledgments}

The authors acknowledge the participating institutions for their help to complete this work.

\section{References}

i. Abdelkareem, B.A., and Eltayeb, M.M., (2015). Seropositivity of toxoplasmosis in Sudanese males blood donors in Military hospital Omdurman/ Sudan. IJB, 6(3), 147-9.

ii. Chandrasena, N., Herath, R., Rupasinghe, N., Samarasinghe, B., Samaranayake, H., Kastuririratne, A., and de Silva, N.R., (2016). Toxoplasmosis awareness, seroprevalence and risk behavior among pregnant women in the Gampaha district, Sri Lanka. Pathog Glob Health, 110(2), 62-7. doi: 10.1080/ 20477724.2016.1173325.

iii. Chong, C.K., Jeong, W., Kim, H.Y., An, D.J., Jeoung, H.Y., Ryu, J.E., Ko, A.R., Kim, Y.J., Hong, S.J., Yang, Z., and Nam, H.W., (2011). Development and clinical evaluation of a rapid serodiagnostic test for toxoplasmosis of cats using recombinant SAG1 antigen. Korean J Parasitol, 49(3), 207-12. doi: 10.3347/ kjp.2011.49.3.207.

iv. El Deeb, H.K., Salah-Eldin, H., Khodeer, S., and Allah, A.A., (2012). Prevalence of Toxoplasma gondii infection in antenatal population in Menoufia Governorate, Egypt. Acta Trop, 124(3), 185-91. doi: 10.1016/ j.actatropica2012.08.005.

v. El-Nahas, H.A., El-Tantawy, N.L., Farag, R.E., and Alsalem, A.M., (2014). Toxoplasma gondii infection among chronic hepatitis C patients: a case-control study. Asian Pac J Trop Med, 7(8):589-93. doi: 10.1016/ S1995-7645(14)60100-0.

vi. Elsheikha, H.M., (2008). Congenital toxoplasmosis: priorities for further health promotion action. Public Health, 122(4), 335-53. DOI:10.1016/j.puhe.2007.08.009.

vii. Elsheikha, H.M., Azab, M.S., Abousamra, N.K., Rahbar, M.H., Elghannam, D.M., and Raafat, D., (2009). Seroprevalence of and risk factors for Toxoplasma gondii antibodies among asymptomatic blood donors in Egypt. Parasitol Res, 104(6), 1471-6. doi: 10.1007/ s00436-009-1350-z.

viii. Hassan, J.S., Ghazi, H.F., and Ahmed, A.H., (2010). Evaluation of Rapid Chromatographic immunoassay with latex agglutination test and (ELISA) for diagnosis of human toxoplamosis. J Fac Med Baghdad, 52(4), 468-70.

ix. Hedman, K., Lappalainen, M., Seppäiä, I., and Mäkelä, O., (1989). Recent Primary Toxoplasma Infection Indicated by a Low Avidity of Specific IgG. J Infect Dis, 159(4), 736-40.

x. Holec-Gasio, L., and Kur, J., (2010). Toxoplasma gondii: Recombinant GRA5 antigen for detection of immunoglobulin G antibodies using enzyme-linked immunosorbent assay. Exp Parasitol, 124(3), 272-8. doi: 10.1016/ j.exppara.2009.10.010.

xi. Huang, X., Xuan, X., Hirata, H., Yokoyama, N., Xu, L., Suzuki N, and Igarashi, I., (2004). Rapid Immunochromatographic Test Using Recombinant SAG2 for Detection of Antibodies against Toxoplasma gondii in Cats. J Clin Microbiol, 42(1), 351-3.

xii. Imam, N.F.A., Azzam, E.A.A., and Attia, and A.A., (2016). Seroprevalence of Toxoplasma gondii among pregnant women in Almadinah Almunawwarah KSA. Journal of Taibah University Medical Sciences, 2016, 11(3):255-9. https:/ / doi.org/ 10.1016/j.jtumed. 2016. 04.004.

xiii. James, B.O., Agbonile, I.O., Okolo, M., Lawani, A.O., and Omoaregba, J.O., (2013). Prevalence of Toxoplasma gondii infection among individuals with severe mental illness in Nigeria: a case control study. Pathog Glob Health, 107(4), 189-93. doi:10.1179/ 2047773213Y.000000 0093.

xiv. Kim, Y.H., Lee, J., Ahn, S., Kim, T.S., Hong, S.J., Chong, C.K., Ahn., H., and Nam, H., (2017). High Seroprevalence of Toxoplasmosis Detected by RDT among the Residents of Seokmo-do (Island) in Ganghwa-Gun, Incheon City, Korea. Korean J Parasitol, 55(1), 9-13. doi: 10.3347/ kjp.2017.55.1.9.

xv. Kim, Y.H., Lee, J.H., Ahn, S.K., Kim, T.S., Hong SJ, Chong CK, Ahn, H., and Nam, H., (2017). Seroprevalence of toxoplasmosis with ELISA and rapid diagnostic test among residents in Gyodong-do, Inchon city, Korea: A four- year follow up. Korean J Parasitol, 55(3):247-54. doi: 10.3347/ kjp.2017.55.3.247

xvi. Kotresha, D., and Noordin, R., (2010). Recombinant proteins in the diagnosis of toxoplasmosis. APMIS,118(8), 529-42. doi: 10.1111/j.1600-0463.2010.02629.x. 
xvii. Lappalainen, M., Koskela, P., Koskiniemi, M., Ammälä, P., Hiilesmaa, V., Teramo, K., Raivio, K.O., Remington, J.S., and Hedman, K., (1993). Toxoplasmosis Acquired during Pregnancy: Improved Serodiagnosis Based on Avidity of IgG. J Infect Dis,167(3), 691-7.

xviii. Liu, Q., Wang, A.D., Huang, S.Y., and Zhu, and X.Q., (2015). Diagnosis of toxoplasmosis and typing of Toxoplasma gondii. Parasit Vectors, 8, 292. DOI:10.1186/ s13071-015-0902-6

xix. Luft, B.J., and Remington, J.S., (1992). Toxoplasmic encephalitis in AIDS. Clin Infect Dis, 15(2), 211-22.

xx. Meek, B., Diepersloot, R.J., van Gool, T., Speijer, D., and Peek, R., (2003). IgM recognition of recombinant Toxoplasma gondii antigens by sera of acutely or latently infected humans. Diagn Microbiol Infect Dis, 45, 45-52. doi.org/ 10.1016/ S0732-8893(02)00476-5.

xxi. Monocada, P.A., and Montoya, J.G., (2012). Toxoplasmosis in the fetus and newborn: an update on prevalence, diagnosis and treatment. Expert Rev Anti infect Ther, 10 (7), 815-28. doi: 10.1586/ eri.12.58.

xxii. Montoya, J.G., and Liesenfeld, O., (2004). Toxoplasmosis. Lancet, 363(9425), 1965-76. DOI:10.1016/ S01406736(04)16412-X

xxiii. Nourollahi, F.S.R., Shad-Del, F., Hosseini, S.M.H., and Dinani, R.S.M., (2004). Standardization and comparison of DotELISA with IFA test for diagnosis of human toxoplasmosis. Iranian Journal of Veterinary Research, University of Shiraz, 5(2),91-6.

xxiv. Ogouyèmi-Hounto, A., Agbayahoun-Chokki, F., de Tove, Y.S.S., Bankole, B.B., de Souza, A.V., Assogba, M., KindeGazard, D., and Massougbodji, A., (2014). Evaluation of a rapid diagnostic test in the diagnosis of toxoplasmosis in pregnant women in Cotonou (Bénin). Bull Soc Pathol Exot, 107(2), 85-9. doi: 10.1007/ s13149-014-0355-8.

xxv. Pappas, G., Roussos, N., and Falagas, M.E., (2009). Toxoplasmosis Snapshot; global status of Toxoplasma gondiiseroprevalence and implications for pregnancy and congenital toxoplasmosis. Int J Parasitol, 39(12), 1385-94. doi: 10.1016/j.jpara.2009.04.003.

xxvi. Robert-Gangneux, F., and Dardé, M.L., (2012). Epidemiology of and diagnostic strategies for toxoplasmosis. Clin Microbiol Rev, 25(2), 264-96. doi: 10.1128/ CMR.05013-11.

xxvii. Roberts, T., Murrell, L.D., and Marks, S., (1994). Economic losses caused by foodborne parasitic disease. Parasitol Today, 10(11), 419-23. https:// doi.org/ 10.1016/ 0169-4758(94)90171-6.

xxviii. Rosso, F., Les, J.T., Agudelo, A., Villalobos, C., Chaves, J.A., Tunubala, G.A., Messa, A., Remington, J.S., and Montoya, J.G., (2008). Prevalence of infection with Toxoplasma gondiiamong pregnant women in Cali, Colombia, South America. Am JTrop Med Hyg, 78, 504-8.

xxix. Simuudic, A.M., (2009). Measures of diagnostic accuracy: Basic definitions, EJIFCC. 19(4), 203-11.

xxx. Song, K.J., Yang, Z., Chong, C., Kim, J.S., Lee, K.C., Kim, T.S., and Nam, H., (2013). A Rapid Diagnostic Test for Toxoplasmosis using Recombinant Antigenic N-terminal Half of SAG1 Linked with Intrinsically Unstructured Domain of GRA2 Protein. Korean J Parasitol. 51(5):503-10. doi: 10.3347/ kjp.2013.51.5.503

xxxi. SYROCOT (Systematic Review on Congenital Toxoplasmosis) study group, Thiébaut, R., Leproust, S., Chêne, G., and Gilbert, R., (2007). Effectiveness of prenatal treatment for congenital toxoplasmosis: a meta-analysis of individual patients' data. Lancet, 369(9556), 115-22. DOI:10.1016/ S0140-6736(07)60072-5

xxxii. Tammam, A.E., Haridy, M.A., Abdellah, A.H., Ahmed, S.R., Fayed, H.M., and Alsammani, M.A., (2013). Seroepidemiology of Toxoplasma gondii infection in women with first trimester spontaneous miscarriage in Qena Governorate, Egypt. J Clin Diagn Res, 7(12), 2870-3. doi: 10.7860/ JCDR/ 2013/ 6480.3780.

xxxiii. Wang, Y.H., Li, X.R., Wang, G.X., Yin, H., Cai, X.P., Fu, B.Q., and Zhang, D.L., (2010). Development of an immunochromatographic strip for the rapid detection of Toxoplasma gondii circulating antigens. Parasitol Int, 6(1), 105-7. DOI:10.1016/ j.parint.2010.11.002

xxxiv. Wilson, M., Remington, J.S., Clavet, C., Varney, G., Press, C., Ware D, and The FDA toxoplasmosis AD HOC working group., (1997), Evaluation of six commercial kits for detection of human immunoglobulin Mantibodies to Toxoplasma gondii. J Clin Microbiol, 35(12), 3112-5.

\section{Appendix}

\begin{tabular}{|c|c|c|c|c|c|c|}
\hline \multirow[t]{3}{*}{ Diagnostic Methods } & \multicolumn{4}{|c|}{ Sample Examined N=188 } & \multirow{2}{*}{\multicolumn{2}{|c|}{ Total Infected }} \\
\hline & \multicolumn{2}{|c|}{ IgG (+ve) } & \multicolumn{2}{|c|}{$\operatorname{Ig} M(+v e)$} & & \\
\hline & No. & $\%$ & No. & $\%$ & No. & $\%$ \\
\hline RDT & 50 & 26.6 & 9 & 4.8 & 50 & 26.6 \\
\hline ELISA & 92 & 48.9 & 31 & 16.5 & 92 & 48.9 \\
\hline Mc Nemar test & \multicolumn{2}{|c|}{$0.001 *$} & & $0.001^{*}$ & \multicolumn{2}{|c|}{$0.001 *$} \\
\hline
\end{tabular}

Table 1: Prevalence of Toxoplasmosis among Mentally Disabled Individuals According to the Diagnostic Methods Used 


\begin{tabular}{|c|c|c|c|c|}
\hline \multirow{2}{*}{ Toxoplasma Specific Antibodies } & \multicolumn{2}{|c|}{ ELISA } & \multicolumn{2}{c|}{ RDT } \\
\cline { 2 - 5 } & No. & $\%$ & No. & $\%$ \\
\hline $\operatorname{IgG}(+\mathrm{ve}) / \operatorname{IgM}(-\mathrm{ve})$ & 61 & 32.45 & 41 & 21.8 \\
\hline $\mathrm{IgG} / \mathrm{IgM}(+\mathrm{ve})$ & 31 & 16.5 & 9 & 4.8 \\
\hline $\mathrm{IgG} / \operatorname{IgM}(-\mathrm{ve})$ & 96 & 51.1 & 138 & 73.4 \\
\hline Total & 188 & 100 & 188 & 100 \\
\hline
\end{tabular}

Table 2: Distribution of Anti-Toxoplasma Specific IGG and IGM Antibodies among Mentally Disabled Individuals by Both Techniques

\begin{tabular}{|c|c|c|c|c|c|c|}
\hline \multirow{2}{*}{ RDT } & \multicolumn{2}{|c|}{ ELISA } & Total Infected & Agreement \% & \multirow{2}{*}{ Kappa } & \multirow{2}{*}{ P } \\
\cline { 2 - 6 } & +ve & -ve & & & \multirow{2}{*}{0.549} & $<0.001^{*}$ \\
\hline +ve & 50 & 0 & 50 & $54.35 \%$ & $100 \%$ & \\
\hline -ve & 42 & 96 & 138 & $77.7 \%$ & & \\
\hline total & 92 & 96 & 188 & & \\
\hline
\end{tabular}

Table 3: Agreement between the RDT and Reference ELISA for Detecting IGG Antibodies in Sera of Mentally Disabled Individuals

\begin{tabular}{|c|c|c|c|c|c|c|}
\hline \multirow{2}{*}{ RDT } & \multicolumn{2}{|c|}{ ELISA } & Total Infected & Agreement \% & Kappa & \multirow{2}{*}{ P } \\
\cline { 2 - 4 } & +ve & -ve & & & \multirow{2}{*}{0.406} & \multirow{2}{*}{$<.001^{*}$} \\
\hline tve & 9 & 0 & 9 & $29.03 \%$ & $100 \%$ & \\
\hline -ve & 22 & 157 & 179 & $88.3 \%$ & & \\
\hline Total & 31 & 157 & 188 & & \\
\hline
\end{tabular}

Table 4: Agreement between RDT and Reference ELISA for Detecting IGMAntibodies in

Sera of Mentally Disabled Individuals

\begin{tabular}{|c|c|c|c|c|c|}
\hline \multirow{2}{*}{ RDT } & \multicolumn{5}{|c|}{ Diagnostic Accuracy Test } \\
\cline { 2 - 6 } & Diagnostic efficiency \% & $\begin{array}{c}\text { Sensitivity \% } \\
(95 \% \text { CI })\end{array}$ & $\begin{array}{c}\text { Specificity \% } \\
(95 \% \text { CI })\end{array}$ & $\begin{array}{c}\text { PPV\% } \\
(95 \% \text { CI })\end{array}$ & $\begin{array}{c}\text { NPV\% } \\
(95 \% \text { CI })\end{array}$ \\
\hline IgG & & 54.35 & 100 & 100 & 69.6 \\
& 77.7 & $(43.7-64.7)$ & $(95.2-100)$ & $(91.1-100)$ & $(61.1-77)$ \\
\hline IgM & & 29.03 & 100 & 100 & 87.7 \\
& 88.3 & $(14.9-48.2)$ & $(79.02-100)$ & $(62.9-100)$ & $(81.8-92)$ \\
\hline
\end{tabular}

Table 5: Diagnostic Parameters of RDT versus ELISA As Diagnostic Gold Standard for Detecting IGG and Igmtoxoplasma Antibodies in Sera of Mentally Disabled Individuals

PPV: Positive Predictive Value

NPV: Negative Predictive Value

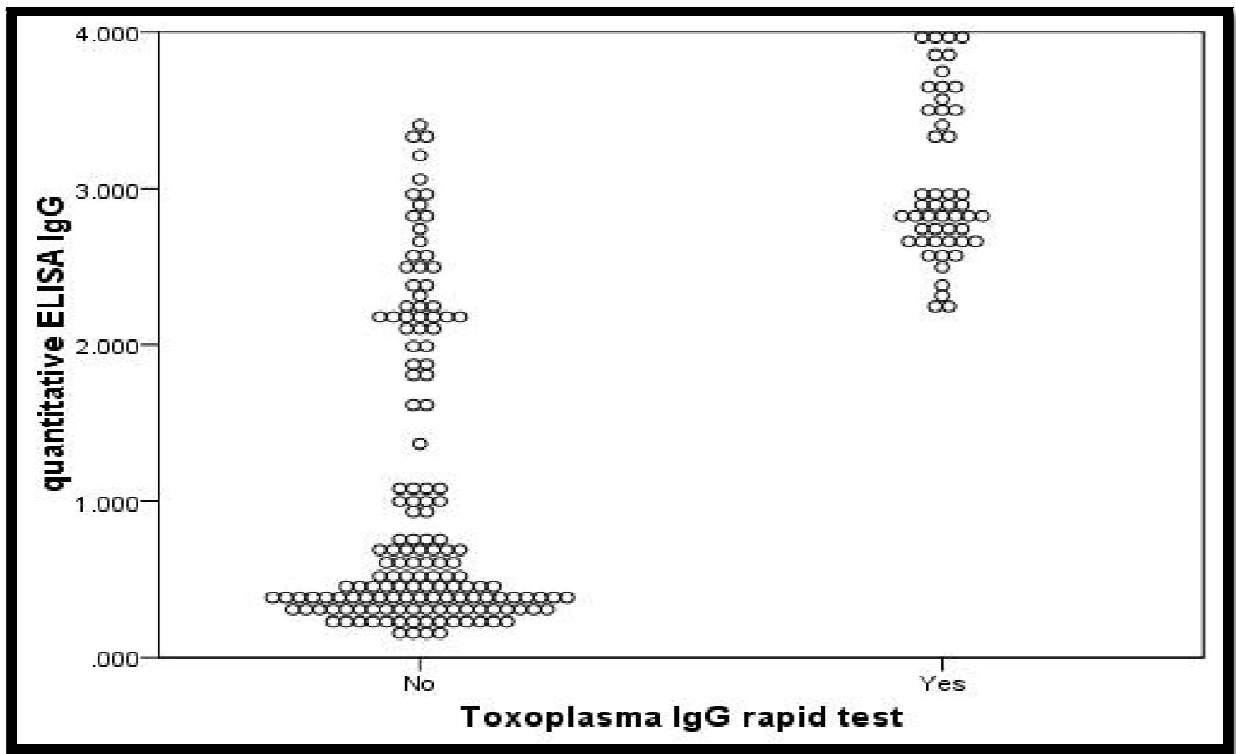

Figure 1: O.D Value of Cut off Calibrator (32IU/ Ml =1.169) 